\title{
Multicenter Optimization and Validation of a 2-Gene mRNA Urine Test for Detection of Clinically Significant Prostate Cancer before Initial Prostate Biopsy
}

\author{
Alexander Haese, Geert Trooskens, ${ }^{*}$ Sandra Steyaert, Daphne Hessels,* Michael Brawer,* \\ Virginie Vlaeminck-Guillem, Alain Ruffion, Derya Tilki, Jack Schalken,* Jack Groskopf* \\ and Wim Van Criekinge* ${ }^{*}+$
}

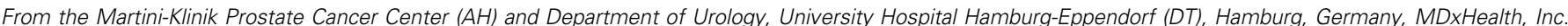
(GT, SS, DH, MB, JG), Irvine, California, Medical Unit of Molecular Oncology and Transfer, Department of Biochemistry and Molecular Biology (VV-G) and Urology

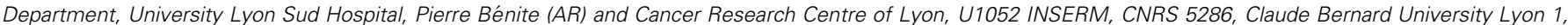
Léon Bérard Centre (VV-G, AR), Lyon, France, Department of Urology, Radboud University Medical Centre (JS), Nijmegen, The Netherlands, and Department of Bioinformatics, Ghent University (WVC), Ghent, Belgium

\begin{abstract}
Abbreviations and Acronyms

DCA $=$ decision curve analysis

$\mathrm{DRE}=$ digital rectal examination

$\mathrm{GG}=$ International Society of

Urological Pathology Grade Group

mpMRI = multiparametric mag-

netic resonance imaging

NPV $=$ negative predictive value

$\mathrm{PCa}=$ prostate cancer

PCPTRC = Prostate Cancer Prevention Trial Risk Calculator, version 2.0

PSA $=$ prostate specific antigen

$\mathrm{PV}=$ prostate volume
\end{abstract}

Accepted for publication April 12, 2019

The corresponding author certifies that, when applicable, a statement(s) has been included in the manuscript documenting institutional review board, ethics committee or ethical review board study approval; principles of Helsinki Declaration were followed in lieu of formal ethics committee approval; institutional animal care and use committee approval, all human subjects provided written informed consent with guarantees of confidentiality; IRB approved protocol number; animal approved project number.

No direct or indirect commercial, personal, academic, political, religious or ethical incentive is associated with publishing this article.

* Financial interest and/or other relationship with MDXHealth.

† Correspondence: Department of Mathematical Modeling, Statistics and Bioinformatics, Ghent University, Coupure Links 653, B-9000 Ghent, Belgium (telephone: +32 0926459 32; e-mail: wim.vancriekinge@gmail.com).
Purpose: A 2-gene, urine based molecular test that combines mRNA biomarkers with clinical factors can risk stratify patients for clinically significant prostate cancer. To ensure the generalizability of assay results we optimized and validated the clinical model for men with serum prostate specific antigen less than $10 \mathrm{ng} / \mathrm{ml}$ who were undergoing initial prostate biopsy.

Materials and Methods: Urine samples were collected from 1,955 men from The Netherlands, France and Germany prior to an initial prostate biopsy and study subjects were divided into training and validation cohorts. Urinary HOXC6 and DLX1 mRNA levels were quantified and RNA results were then combined with other risk factors in a clinical model optimized to detect ISUP (International Society of Urological Pathology) Grade Group 2 or greater prostate cancer in men with prostate specific antigen less than $10 \mathrm{ng} / \mathrm{ml}$. Results in the validation cohort were compared with the PCPTRC (Prostate Cancer Prevention Trial Risk Calculator), version 2.0.

Results: The optimal clinical model included urinary HOXC6 and DLX1 mRNA levels, patient age, digital rectal examination and prostate specific antigen density (serum prostate specific antigen/prostate volume). In the 715 validation cohort subjects with prostate specific antigen less than $10 \mathrm{ng} / \mathrm{ml}$ the AUC was 0.82 with $89 \%$ sensitivity, $53 \%$ specificity and $95 \%$ negative predictive value. The PCPTRC AUC was 0.70 . The full validation cohort of 916 men including all prostate specific antigen levels yielded an AUC of 0.85 with 93\% sensitivity, $47 \%$ specificity and $95 \%$ negative predictive value. The PCPTRC AUC was 0.76 .

Conclusions: The 2-gene based urine assay, which is optimized for biopsy naïve patients with serum prostate specific antigen less than $10 \mathrm{ng} / \mathrm{ml}$, demonstrated high sensitivity and negative predictive value to detect clinically significant prostate cancer. These data support using the test to help guide initial prostate biopsy decisions.

Key Words: prostatic neoplasms; RNA, messenger; prostate specific antigen; biopsy; risk
https://doi.org/10.1097/JU.0000000000000293 Vol. 202, 256-263, August 2019 Printed in U.S.A. 
THERE is a persistent unmet diagnostic need for better methods to identify clinically significant PCa. Despite substantial evidence of the survival benefit associated with the treatment of intermediate and high risk, early stage disease, ${ }^{1-4}$ the low specificity of PSA screening has led to excess prostate biopsies and over detection of lower risk cancers ${ }^{5,6}$ treatment of which lacks proven benefit. ${ }^{7,8}$ Prostate biopsy procedures and interventional cancer treatment may carry significant risks, especially in patients with comorbidities. $^{9,10} \mathrm{PCa}$ specific biomarkers used in conjunction with PSA and other clinical risk factors ${ }^{11}$ can improve the detection of $\mathrm{PCa}$ with specific aggressive features such as Gleason score 7 (GG2) or higher grade, thereby helping to maximize the cancer survival benefit while also avoiding prostate biopsies which would detect indolent or no PCa.

A 2-gene urine based molecular test targeting mRNA known to be overexpressed in aggressive $\mathrm{PCa}$, the cell proliferation gene HOXC6 and the progression gene DLX1, has been validated for the detection of GG2 and higher $\mathrm{PCa} .{ }^{12,13}$ Urinary HOXC6 and DLX1 mRNA levels are measured following DRE and the RNA results are combined with clinical risk factors to determine the individualized patient risk of GG2 or higher PCa histopathology at a subsequent biopsy. ${ }^{13}$ The 2-gene test was clinically validated for the detection of GG2 or greater $\mathrm{PCa}$ in a prospective multicenter cohort of 386 subjects, including 342 (89\%) undergoing initial biopsy and $44(11 \%)$ with prior PCa negative biopsies, and the test had $98 \%$ NPV. ${ }^{13}$ Analytical validation studies have demonstrated the robustness and reproducibility of the 2-gene urine test results. ${ }^{14}$ Risk scores have also been associated with the mpMRI outcome, suggesting a potential role for identifying the patients most likely to benefit from mpMRI or for risk stratification following indeterminate mpMRI results. ${ }^{15}$

The cost-effectiveness of the 2-gene test in men with elevated PSA was assessed in European and American modeling studies. ${ }^{16,17}$ In each study using the 2-gene test decreased prostate biopsy with its associated over diagnosis and/or overtreatment compared to the current standard of care, which resulted in cost savings and increased quality adjusted life years per patient. $^{16,17}$ In addition, the impact on initial biopsy decision making was demonstrated in a multicenter study of community urology practices in the United States. ${ }^{18}$ The initial biopsy rate was fivefold higher in test positive vs negative patients.

In this multicenter study we optimized the 2-gene urinary biomarker based clinical model in men with PSA levels less than $10 \mathrm{ng} / \mathrm{ml}$ who were undergoing initial prostate biopsy. We then validated its clinical performance in all subjects regardless of serum PSA level.

\section{MATERIALS AND METHODS}

\section{Study Population}

The study population consisted of 1,955 sequentially enrolled men, including 750 from Radboud University Medical Centre, Nijmegen, The Netherlands; 762 from Lyon Sud Hospital, Lyon, France; and 443 from the Martini-Klinik Prostate Cancer Center, Hamburg, Germany. These men underwent an initial prostate biopsy for suspected PCa between December 2007 and December 2014. All subjects underwent extended 10 to 12-core transrectal ultrasound guided biopsy. PV was determined by ultrasound at biopsy. In 295 of the 1,955 subjects mpMRI was done prior to biopsy to help target supplemental cores during the transrectal ultrasound guided procedure. None of these men underwent mpMRI fusion biopsy.

Subjects were divided into 1,039 in the training cohort and 916 in the validation cohort. The Dutch training and validation cohorts were previously described. ${ }^{13}$ The French and German cohorts were evenly split by randomization. Table 1 shows a summary of study population demographic and clinical characteristics in the 805 and 715 men in the training and validation cohorts, respectively, with PSA less than $10 \mathrm{ng} / \mathrm{ml}$ as well as the entire cohort.

Study exclusion criteria were a prior diagnosis of $\mathrm{PCa}$, medical therapy known to affect serum PSA levels within 6 months prior to urine sample collection, such as finasteride (Proscar ${ }^{\circledR}$ or Propecia ${ }^{\circledR}$ ), dutasteride (Avodart ${ }^{\circledR}$ ), antiandrogen therapies (Lupron ${ }^{\circledR}$ or Eligard ${ }^{\circledR}$ ), and invasive treatment of $\mathrm{BPH}$ within 6 months of urine sample collection.

\section{Sample Collection and Processing}

Prior to biopsy first catch urine was collected after a standardized DRE consisting of 3 sweeps per prostatic lobe. The urine was mixed with a RNA preservative $\left(\right.$ Hologic $\left.{ }^{\circledR}\right)$ and stored at $-70 \mathrm{C}$. Urine samples were tested elsewhere with 2-gene reverse transcriptase-polymerase chain reaction. Laboratory technicians were blinded to biopsy outcomes.

Briefly, HOXC6 and DLX1 mRNA were quantified using reverse transcriptase-polymerase chain reaction and then normalized for prostate RNA recovery using urinary KLK3 mRNA levels. KLK3 is the gene that encodes for PSA. The normalized HOXC6 and DLX1 levels were combined into a single RNA value, which was then used with other risk factors in a clinical model, resulting in a continuous risk score of -6 to 6 . To report results the risk score was then converted to the percent likelihood that subsequent biopsy would identify GG2 or greater PCa. ${ }^{13,14}$

\section{Statistical Analysis}

The clinical model was designed to discriminate GG2 or higher PCa from GG1 or no PCa at biopsy. Model variables included the urinary RNA value, patient age, PSA density calculated as serum PSA/prostate volume and the DRE result (normal or suspicious). Although the AUC often serves as a metric for clinical performance, only a small part of the ROC curve is relevant in the initial prostate biopsy setting, in which the patient risk 
Table 1. Subject demographic and clinical characteristics in training and validation cohorts

\begin{tabular}{|c|c|c|c|c|c|c|c|c|}
\hline \multirow{5}{*}{$\begin{array}{l}\text { No. subjects } \\
\text { Median age (IQR) } \\
\text { Median ng/ml serum PSA (IQR) } \\
\text { No. DRE result (\%): }\end{array}$} & \multicolumn{4}{|c|}{ PSA Less Than $10 \mathrm{ng} / \mathrm{ml}$} & \multicolumn{4}{|c|}{ All Subjects } \\
\hline & \multicolumn{2}{|c|}{ Training } & \multicolumn{2}{|c|}{ Validation } & \multicolumn{2}{|c|}{ Training } & \multicolumn{2}{|c|}{ Validation } \\
\hline & \multicolumn{2}{|c|}{805} & \multicolumn{2}{|c|}{715} & \multicolumn{2}{|c|}{1,039} & \multicolumn{2}{|c|}{916} \\
\hline & \multicolumn{2}{|c|}{$\begin{array}{cc}64 & (59-68) \\
5.5 & (4.1-6.9)\end{array}$} & \multicolumn{2}{|c|}{$\begin{array}{cr}64 & (59-69) \\
5.4 & (4.1-7.2)\end{array}$} & \multicolumn{2}{|c|}{$\begin{array}{cr}64 & (59-69) \\
6.2 & (4.6-9.4)\end{array}$} & \multicolumn{2}{|c|}{$\begin{array}{cr}65 & (60-70) \\
6.4 & (4.5-9.2)\end{array}$} \\
\hline & \\
\hline Normal & 636 & (79) & 585 & (82) & 758 & (73) & 706 & (77) \\
\hline Suspicious & 168 & (21) & 124 & (17) & 280 & (27) & 205 & (22) \\
\hline Yes & 126 & (16) & 110 & (15) & 148 & (14) & 130 & (14) \\
\hline No & 648 & (80) & 499 & (70) & 845 & (81) & 632 & (69) \\
\hline Not available & 31 & (4) & 106 & (15) & 46 & (4) & 154 & (17) \\
\hline \multicolumn{9}{|l|}{ Biopsy outcome (\%): } \\
\hline No PCa & 455 & $(56.5)$ & 388 & (54.3) & 518 & (49.9) & 449 & $(49.0)$ \\
\hline GG1 & 196 & (24.3) & 175 & (24.5) & 239 & (23.0) & 209 & (22.8) \\
\hline
\end{tabular}

associated with false-negative results is higher than that of false-positive findings. ${ }^{19}$ Therefore, we defined the cost function to optimize the model as the average falsepositive rate (1 - specificity) of the ROC curve interval between $93 \%$ and $96 \%$ sensitivity. The coefficients of the 4 variables were exhaustively determined by minimizing the cost function. To allow for cases in which PV was not available we generated an alternate model to maintain similar sensitivity and NPV by allowing variable specificity. In the absence of PV PSA density was replaced by serum PSA as a stand-alone variable.

Statistical analyses were performed with $\mathrm{R}$, version 3.4.0 (R Foundation for Statistical Computing, Vienna, Austria). The clinical performance of RNA biomarkers, clinical variables and models was assessed based on the ROC AUC. The 95\% CI and AUC comparisons were determined by the DeLong method as implemented in the $\mathrm{R}$ package pROC. ${ }^{20,21}$ The Mann-Whitney test was applied to assess differences in quantitative measurements. The PCPTRC was used for comparison. DCA was performed in $\mathrm{R}$ as previously described. ${ }^{22}$ Calibration curves were generated in $\mathrm{R}$ using the val.prob function. ${ }^{21,23}$

\section{RESULTS}

\section{Combining Risk Factors to Detect High Grade Prostate Cancer}

Clinical models were developed using 805 training cohort subjects with serum PSA less than $10 \mathrm{ng} / \mathrm{ml}$ (table 1). This yielded an AUC of $0.80(95 \%$ CI $0.76-0.84)$. At the optimal risk score cutoff of -2.8 sensitivity was $93 \%$, specificity was $52 \%$ and NPV was $96 \%$. Figure 1 shows the relationship between the risk score and the likelihood of GG2 or higher $\mathrm{PCa}, \mathrm{GG} 1 \mathrm{PCa}$ and no PCa at biopsy using data on the full sample set of 1,955 primary biopsies. Higher risk scores were associated with an increased likelihood of GG2-5 PCa at biopsy, providing an individualized risk assessment to assist with biopsy decision making.

\section{Clinical Validation}

In the training cohort of 805 men with PSA less than $10 \mathrm{ng} / \mathrm{ml}$ the AUC was $0.80(95 \%$ CI $0.76-0.84)$. At the optimal risk score cutoff of -2.8 sensitivity was $93 \%$, specificity was $52 \%$ and NPV was $96 \%$. The optimized clinical model was applied to the 715 validation cohort subjects with serum PSA less than $10 \mathrm{ng} / \mathrm{ml}$. This yielded an AUC of 0.82 (95\% CI 0.79-0.86), 89\% sensitivity, 53\% specificity and 95\% NPV. When PV was omitted from the model, sensitivity was $87 \%$, specificity was $38 \%$ and NPV was $92 \%$.

Because many clinicians use suspicious DRE results as a stand-alone indication for biopsy, we also evaluated performance in subjects with normal DRE results. Table 2 summarizes performance

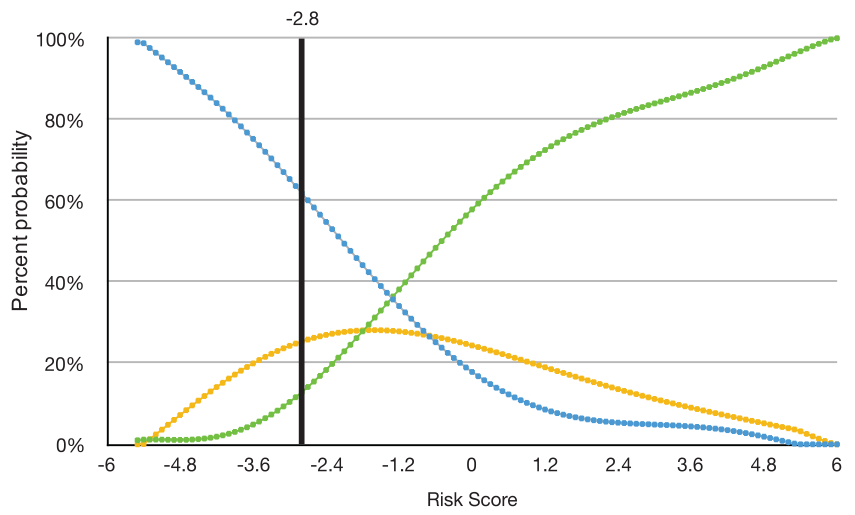

Figure 1. Percent probability of GG2 or greater (green curve), no $\mathrm{PCa}$ (blue curve) and GG1 PCa (yellow curve) at biopsy in relation to risk score. Vertical line at risk score -2.8 represents cutoff point of overall positive vs negative result. 
Table 2. Optimized clinical model performance characteristics in validation cohort stratified by serum PSA and digital rectal examination results

\begin{tabular}{|c|c|c|c|c|}
\hline & \multicolumn{2}{|c|}{ PSA Less Than $10 \mathrm{ng} / \mathrm{ml}$} & \multicolumn{2}{|c|}{ All PSA Results } \\
\hline & All DRE Results & Normal DRE Results & All DRE Results & Normal DRE Results \\
\hline No. subjects & 715 & 591 & 916 & 712 \\
\hline$\%$ Sensitivity & 89 & 84 & 93 & 89 \\
\hline$\%$ Specificity & 53 & 57 & 47 & 41 \\
\hline$\%$ Pos predictive value & 34 & 29 & 43 & 33 \\
\hline
\end{tabular}

Negative predictive value remained constant at $95 \%$ across all patient subgroups.

characteristics in the validation cohort, stratified by PSA (PSA less than $10 \mathrm{ng} / \mathrm{ml}$ or all values) and the DRE result. For all conditions the NPV was $95 \%$. The optimized model was also applied to the validation cohort from the previous clinical validation study. ${ }^{13}$ The AUC was 0.88 (0.84-0.92), sensitivity was $98 \%$, specificity was $42 \%$ and NPV was $98 \%$.

Figure 2 shows ROC curves of the optimized clinical model and the PCPTRC in the subject groups. In validation cohort subjects with PSA less than $10 \mathrm{ng} / \mathrm{ml}$ the AUC was 0.82 (95\% CI 0.79-0.86) compared to the PCPTRC AUC of 0.70 (95\% CI 0.65-0.74, p <0.001). In the entire validation cohort regardless of PSA level the optimized clinical model AUC was 0.85 (95\% CI $0.83-0.88$ ), with $93 \%$ sensitivity, $47 \%$ specificity and 95\% NPV while the PCPTRC AUC was 0.76 (95\% CI $0.72-0.80, \mathrm{p}<0.001$ ).

To further characterize the optimized model we analyzed the incremental benefit of the urinary biomarkers relative to clinical risk factors and assessed the model calibration. Figure 3 shows urinary HOXC6 and DLX1 mRNA levels normalized to KLK3 as the prostate specific control in validation cohort subjects with no PCa, GG1 PCa, or GG2 or greater $\mathrm{PCa}$ at biopsy. For each mRNA the relative level was significantly higher in men diagnosed with GG2 or greater PCa vs GG1 PCa $(p<0.0001)$.

DCA was performed using the optimized model with and without HOXC6 and DLX1 mRNA measurements (fig. 4). The DCA showed a net benefit of the full model compared to the no RNA model to detect GG2 or higher PCa while avoiding excess biopsies, particularly at lower risk thresholds. Results were similar in men with PSA less than $10 \mathrm{ng} / \mathrm{ml}$ and in the full validation cohort (fig. 4).

Figure 5 shows calibration plots of the risk score and PCPTRC in the full validation cohort of 916 subjects. The optimized model showed good

B

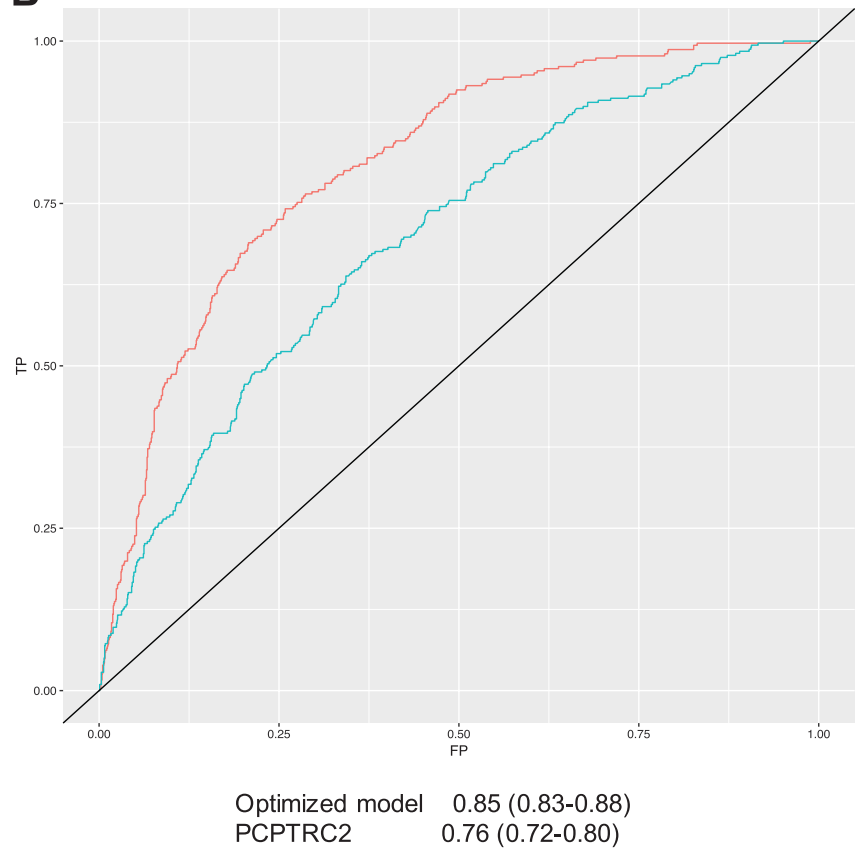

Figure 2. ROC curves of optimized clinical model (red curves) and PCPTRC (blue curves) in 715 validation cohort subjects with PSA less than $10 \mathrm{ng} / \mathrm{ml}(A)$ and full validation cohort of 916 with all PSA levels $(B)$.

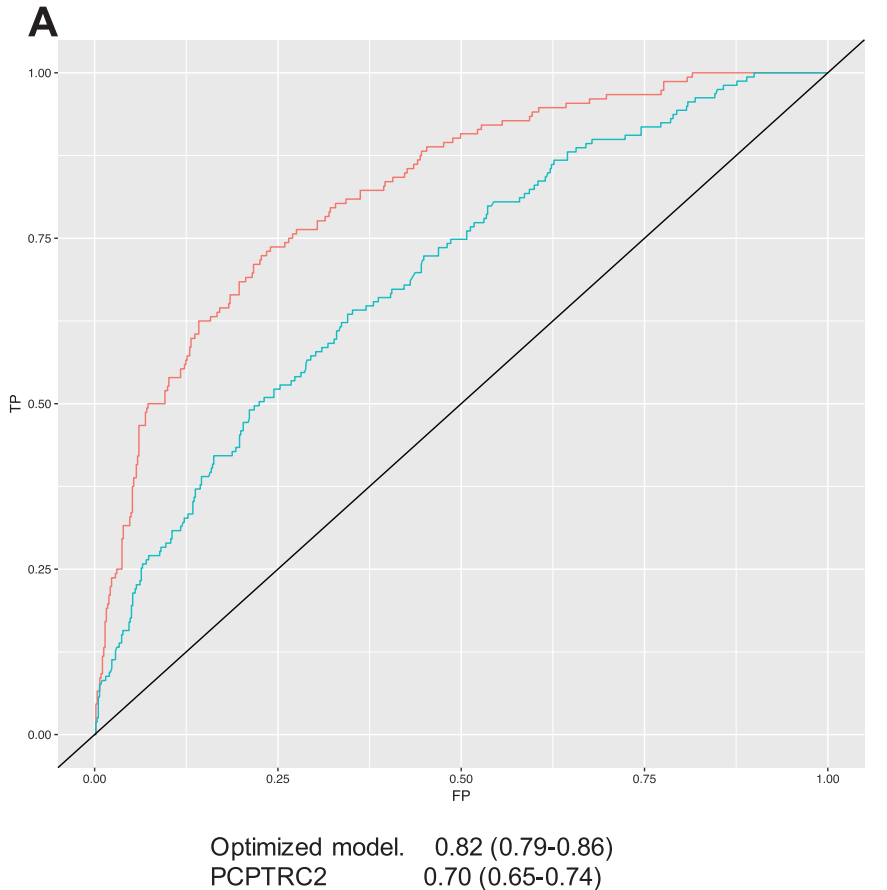


A

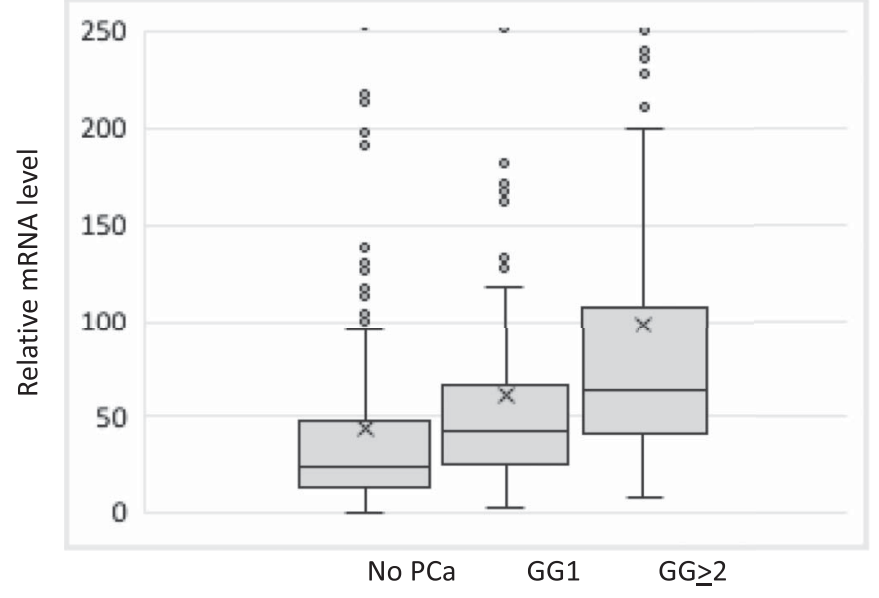

B

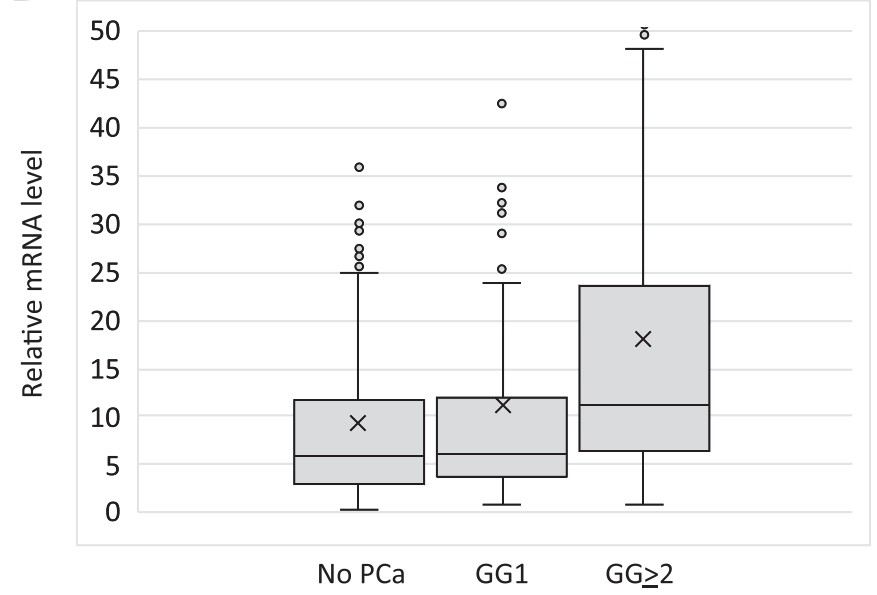

Figure 3. Box plots of urinary HOXC6 $(A)$ and DLX1 $(B)$ mRNA in subjects with no PCa, low grade PCa or clinically significant PCa at biopsy. Horizontal line indicates median. $x$ indicates average.

calibration with a slight trend toward overestimating risk at probabilities below approximately $25 \%$ and underestimating risk at higher probabilities (fig. 5, A). As a point of reference the risk score threshold used to determine overall positive vs negative assay results and determine performance characteristics (table 2) corresponded to a probability of $12 \%$. In contrast, the PCPTRC consistently underestimated risk in the validation cohort (fig. 5, B).

To assess the potential impact of the optimized model on patient treatment the validation cohort of 715 men with PSA less than $10 \mathrm{ng} / \mathrm{ml}$ was evaluated when assuming that assay results were the sole determinant for the biopsy decision on study. Based on the patients with negative test results 314 of all 715 biopsies (44\%) and 297 of 563 biopsies (53\%) which identified GG1 or no PCa would have been avoided or delayed. In patients with negative test results who were found to have $\mathrm{PCa}$ at biopsy 61 of 175 (35\%) with GG1 PCa, 12 of 97 (12\%) with GG2, 4 of $27(15 \%)$ with GG3, 1 of $17(5.9 \%)$ with GG4 and 0 of $11(0 \%)$ with GG5 may have had a delayed diagnosis. In the overall validation cohort 17 of 715 men (2.4\%) had negative test results and were diagnosed with GG2 or greater PCa at biopsy.

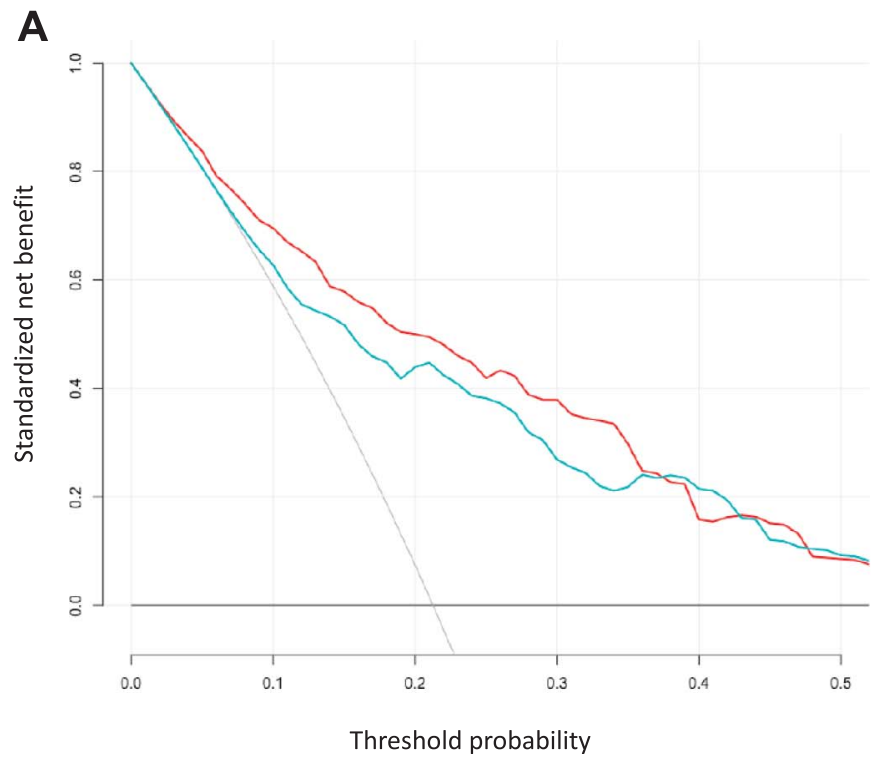

B

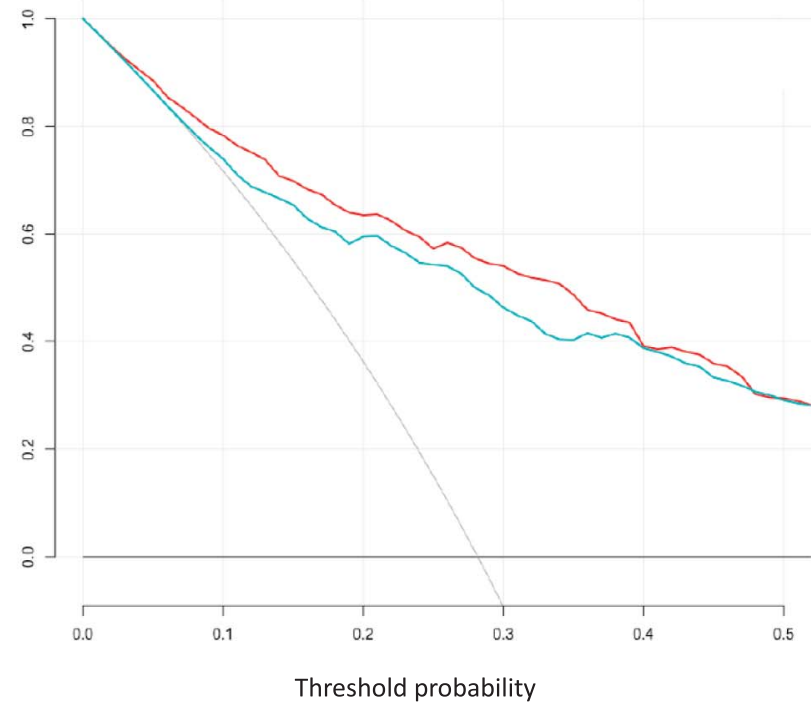

Figure 4. Decision curve analysis of full clinical model (red curves) vs clinical model without RNA markers (blue curves) in validation cohort subjects with PSA less than $10 \mathrm{ng} / \mathrm{mL}(A)$ and in full validation cohort $(B)$. Gray curve: all subjects have GG2 or higher PCa, horizontal line: no subjects have GG2 or higher PCa. 
A

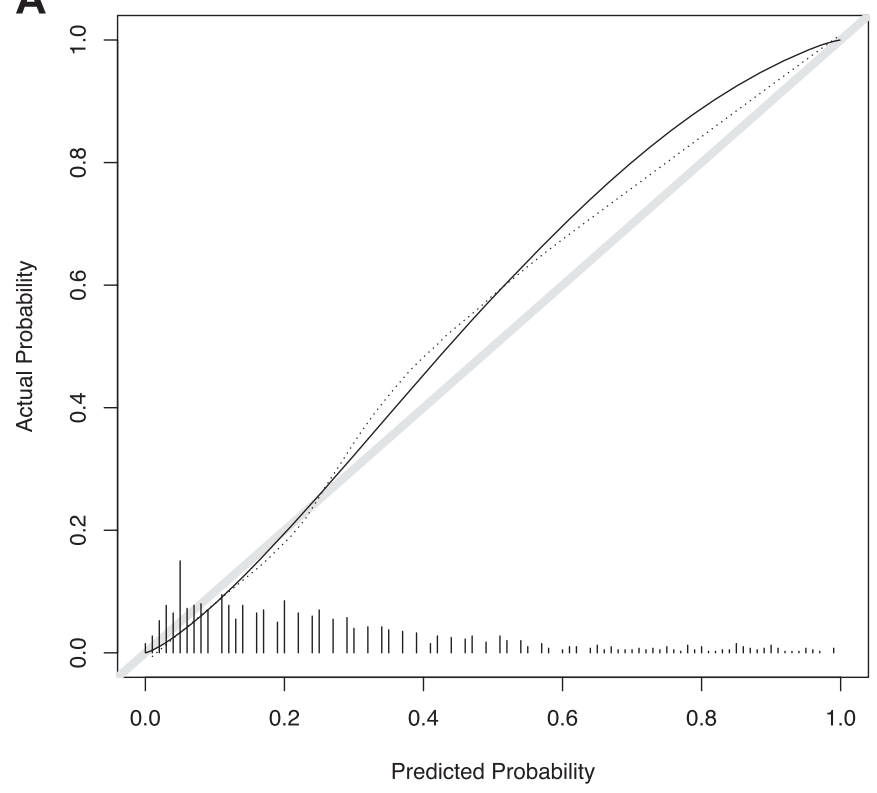

B

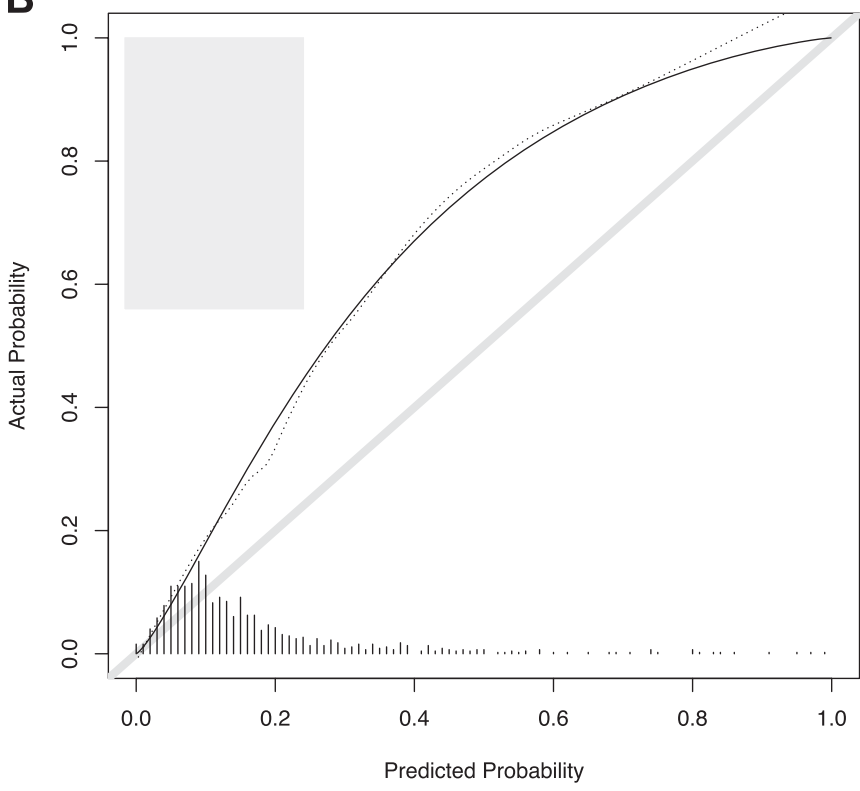

Figure 5. Calibration plots of risk score $(A)$ and PCPTRC $(B)$ in validation cohort. Gray curve indicates ideal. Black curve indicates logistic calibration. Dotted curve indicates nonparametric data.

\section{DISCUSSION}

Accurately identifying clinically significant $\mathrm{PCa}$ remains one of the key unmet needs in the diagnosis and management of this pervasive disease. An approach to this dilemma is to use $\mathrm{PCa}$ specific biomarkers which can be combined with clinical risk factors to improve the detection of GG2 and higher PCa. We previously described the development and validation of the 2-gene urinary biomarker based clinical model to discriminate GG2 and higher PCa from GG1 and benign disease in men undergoing prostate biopsy. ${ }^{13}$ In this study we confirmed and expanded these findings in a larger, multinational population of men who were undergoing an initial prostate biopsy with an emphasis on patients with PSA less than $10 \mathrm{ng} / \mathrm{ml}$.

The clinical model was optimized to maintain high sensitivity and NPV to detect GG2 or greater $\mathrm{PCa}$ in the initial biopsy setting. In the validation cohort of 715 subjects with PSA less than $10 \mathrm{ng} / \mathrm{ml}$ the AUC was 0.82 , sensitivity was $89 \%$, specificity was $53 \%$ and NPV was $95 \%$. If the 2-gene test had been used for the initial biopsy decision, $53 \%$ of excess biopsies (ie biopsies which would have identified GG1 or no cancer) could have been avoided and a diagnosis of clinically significant PCa may have been delayed in $5 \%$ of patients.

Because PV is not always available in biopsy naïve men, the model was designed to compensate and yielded a NPV of $92 \%$ when this parameter was not included. In all 916 validation cohort subjects with any PSA level sensitivity was $93 \%$, specificity was $47 \%$ and NPV was $95 \%$. The NPV of $95 \%$ was maintained when analysis was limited to men with normal DRE results, who therefore might be more likely to benefit from additional testing. Importantly, when the optimized model was applied to the validation cohort in the initial validation study, ${ }^{13}$ results were equivalent to previously reported findings with 98\% NPV. Taken together these results demonstrate the robustness of the clinical model across different patient cohorts.

Characterization of the optimized model confirmed that HOXC6 and DLX1 mRNA measurements provide a significant improvement in test performance compared to clinical risk factors alone. Urinary levels of the individual mRNAs were significantly associated with clinically significant $\mathrm{PCa}$, consistent with previous reports. ${ }^{13,14}$ DCA demonstrated net benefit of the RNA biomarkers, particularly at lower risk thresholds where the test may be most useful in clinical practice. Finally, the optimized clinical model was found to be well-calibrated for predicting the probability of detecting GG2 or higher PCa at biopsy.

Several other options are available to clinicians to help guide initial biopsy decisions. Increasingly mpMRI is performed for PCa detection. However, a recent meta-analysis highlighted mpMRI variability with $64 \%$ to $88 \%$ NPVs,${ }^{24}$ lower than in our report. In addition, the AUA (American Urological Association) currently recommends mpMRI only in the repeat biopsy setting. ${ }^{25}$

Blood based PSA derivative assays and other urinary markers have shown potential to improve the prediction of the initial biopsy outcome. The 
results of this clinical validation compare favorably with those of published studies using these other methodologies. ${ }^{11}$

The strengths of this multicenter validation study include the relatively large training and validation cohorts, and the assessment of clinical performance in all patients with an initial biopsy using a model optimized for men with PSA less than $10 \mathrm{ng} / \mathrm{ml}$. Limitations include the fact that the study was retrospective and there was a lack of racial/ ethnic background information on the study population, which was presumed to be primarily Caucasian. This likely does not reflect the diversity of potential patients who undergo an initial biopsy in the United States.

\section{CONCLUSIONS}

The 2-gene urine biomarker based assay was optimized for biopsy naïve patients with serum PSA less than $10 \mathrm{ng} / \mathrm{ml}$ and clinically validated in a multicenter study. The 2-gene test demonstrated high sensitivity and NPV to detect GG2 or greater PCa with the potential to improve the detection of clinically significant disease by avoiding approximately half of excess prostate biopsies. These data support the use of this test to help guide initial prostate biopsy decisions.

\section{ACKNOWLEDGMENT}

Reverse transcriptase-polymerase chain reaction was performed at MDxHealth, Nijmegen, The Netherlands.

\section{REFERENCES}

1. Bill-Axelson A, Holmberg $L$, Garmo $H$ et al: Radical prostatectomy or watchful waiting in early prostate cancer. N Engl J Med 2014; 370: 932.

2. Bolla M, Gonzalez D, Warde P et al: Improved survival in patients with locally advanced prostate cancer treated with radiotherapy and goserelin. N Engl J Med 1997; 337: 295

3. D'Amico AV, Manola J, Loffredo $M$ et al: 6month androgen suppression plus radiation therapy vs radiation therapy alone for patients with clinically localized prostate cancer: a randomized controlled trial. JAMA 2004; 292: 821.

4. Widmark A, Klepp 0, Solberg A et al: Endocrine treatment, with or without radiotherapy, in locally advanced prostate cancer (SPCG-7/SFU03): an open randomised phase III trial. Scandinavian Prostate Cancer Group Study 7 and Swedish Association for Urological Oncology 3. Lancet 2009; 373: 301

5. Andriole GL, Crawford ED, Grubb RL 3rd et al; PLCO Project Team: mortality results from a randomized prostate-cancer screening trial. N Engl J Med 2009; 360: 1310.

6. Schröder FH, Hugosson J, Roobol MJ et al: Screening and prostate-cancer mortality in a randomized European study. ERSPC Investigators. N Engl J Med 2009; 360: 1320.

7. Wilt TJ, Brawer MK, Jones KM et al: Radical prostatectomy versus observation for localized prostate cancer. Prostate Cancer Intervention versus Observation Trial (PIVOT) Study Group. N Engl J Med 2012; 367: 203.

8. Wilt TJ, Jones KM, Barry MJ et al: Follow-up of prostatectomy versus observation for early prostate cancer. N Engl J Med 2017; 377: 132.
9. Loeb S, Vellekoop A, Ahmed HU et al: Systematic review of complications of prostate biopsy. Eur Urol 2013; 64: 876.

10. Draisma G, Etzioni R, Tsodikov A et al: Lead time and overdiagnosis in prostate-specific antigen screening: importance of methods and context. J Natl Cancer Inst 2009; 101: 374.

11. Dani $\mathrm{H}$ and Loeb $\mathrm{S}$ : The role of biomarkers in undiagnosed men. Curr Opin Urol 2017; 27: 210.

12. Leyten GH, Hessels D, Smit FP et al: Identification of a candidate gene panel for the early diagnosis of prostate cancer. Clin Can Res 2015; 21: 3061 .

13. Van Neste L, Hendriks RJ, Dijkstra $S$ et al: Detection of high-grade prostate cancer using a urinary molecular biomarker-based risk score. Eur Urol 2016; 70: 740.

14. Hessels D, de Jong $H$, Jannink SA et al: Analytical validation of an mRNA-based urine test to predict the presence of high-grade prostate cancer. Trans Med Commun 2017; 2: 5 .

15. Hendriks RJ, van der Leest MM, Dijkstra $S$ et al: A urinary biomarker-based risk score correlates with multiparametric MRI for prostate cancer detection. Prostate 2017; 77: 1401.

16. Dijkstra S, Govers TM, Hendriks RJ et al: Costeffectiveness of a new urinary biomarker-based risk score compared to standard of care in prostate cancer diagnostics-a decision analytical model. BJU Int 2017; 120: 659.

17. Govers T, Caba L and Resnick MJ: Costeffectiveness of SelectMDx in prostate cancer risk assessment. J Urol 2018; 200: 1221.

18. Shore N, Hafron J, Langford $T$ et al: Urinary molecular biomarker test impacts prostate biopsy decision-making in clinical practice. Urol
Pract 2018; available at https://doi.org/10.1016/ j.urpr.2018.09.002.

19. Swamidass SJ, Azencott CA, Daily K et al: A CROC stronger than ROC: measuring, visualizing and optimizing early retrieval. Bioinformatics 2010; 26: 1348.

20. DeLong ER, DeLong DM and Clarke-Pearson DL: Comparing the areas under two or more correlated receiver operating characteristic curves: a nonparametric approach. Biometrics 1988; 144: 837.

21. Robin $X$, Turck $N$, Hainard $A$ et al: pROC: an open-source package for $\mathrm{R}$ and $\mathrm{S}+$ to analyze and compare ROC curves. BMC Bioinformatics 2011; 12: 77

22. Vickers AJ and Elkin EB: Decision curve analysis, a novel method for prediction models. Med Decis Making 2006; 26: 565.

23. Harrell FE, Lee KL and Mark DB: Multivariable prognostic models: issues in developing models, evaluating assumptions and adequacy, and measuring and reducing errors. Stat Med 1996; 15: 361.

24. Moldovan PC, Van den Broeck T, Sylvester R et al: What is the negative predictive value of multiparametric magnetic resonance imaging in excluding prostate cancer at biopsy? A systematic review and meta-analysis from the European Association of Urology Prostate Cancer Guidelines Panel. Eur Urol 2017; 72: 250.

25. Rosenkrantz AB, Verma S, Choyke P et al: Prostate magnetic resonance imaging and magnetic resonance imaging targeted biopsy in patients with a prior negative biopsy: a consensus statement by AUA and SAR. J Urol 2016; 196: 1613. 


\section{EDITORIAL COMMENT}

In the United States a shotgun approach to screening and treatment led to a $52 \%$ reduction in age adjusted prostate cancer specific mortality but this was tempered by the pandemic of men who never needed to be diagnosed, let alone treated. This mRNA urine test (SelectMDx ${ }^{\circledR}$ ) is scientifically sound, now has 2 validation studies and is capable of decreasing the biopsy rate by $40 \%$ to $50 \%$ while delaying the diagnosis in $2 \%$ to $5 \%$ of men known to have GG2 or greater (Gleason 7 or greater cancer). Interestingly, similar to other biomarkers, no consistent relationship was observed between the biomarker risk score and GG1 (Gleason 6) (fig. 1 in article), further evidence corroborating its inert nature.

About 10 biomarkers are available or in development with similar intent, of which most are costly. It is a busy clinical space but collectively a much welcomed advance. At a bare minimum when urologists screen for prostate cancer, they should capitalize on the powerful information embedded in inexpensive tests such as total PSA, percent free PSA, PSA density and digital rectal examination. Use of secondary markers (magnetic resonance imaging, or urine, blood or tissue based markers) is data based and sensible but pricey. Reflex use in all men is mindless and should be discouraged. When used appropriately, added value is provided, particularly when scores are low or high.

Scott Eggener Departments of Surgery and Radiology University of Chicago Medicine Chicago, Illinois 